\title{
Lenvatinib Promotes the Antitumor Effect of Doxorubicin in Anaplastic Thyroid Cancer
}

This article was published in the following Dove Press journal: OncoTargets and Therapy

\author{
$\mathrm{Xi} \mathrm{Su}^{\prime}$ \\ Jiaxin Liu' \\ Haihong Zhang' \\ Qingqing $\mathrm{Gu}^{\prime}$ \\ Xinrui Zhou' \\ Meiju Ji ${ }^{2}$ \\ Demao Yao $\mathbb{( D}^{3}$
}

'Key Laboratory for Tumor Precision Medicine of Shaanxi Province, Department of Endocrinology, The First Affiliated Hospital of Xi'an Jiaotong University, Xi'an 7I006I, People's Republic of China;

${ }^{2}$ Center for Translational Medicine, The First Affiliated Hospital of Xi'an Jiaotong University, Xi'an 71006I, People's Republic of China; ${ }^{3}$ Department of Geriatric Surgery, The First Affiliated Hospital of X'an Jiaotong University, Xi'an 71006I, People's Republic of China
Correspondence: Demao Yao Department of Geriatric Surgery, The First Affiliated Hospital of Xi'an Jiaotong University, No. 266, Yanta West Road, Xi'an 71006I, People's Republic of China $\mathrm{Tel} / \mathrm{Fax}+86$ |399|953|23 Emailydm_723@163.com

Meiju ji

Center for Translational Medicine, The First Affiliated Hospital of Xi'an Jiaotong University, No. 266, Yanta West Road,

Xi'an 7I006I, People's Republic of China

$\mathrm{Tel} / \mathrm{Fax}+862985323259$

Email mjji0409@I63.com
Purpose: Anaplastic thyroid cancer (ATC) is a kind of rare thyroid cancer with very poor prognosis. Doxorubicin has been approved in ATC treatment as a single agent, but monotherapy still shows no improvement of the total survival in advanced ATC. Lenvatinib was investigated with encouraging results in treating patients with radioiodine-refractory differentiated thyroid cancer (DTC). However, antitumor efficacy of combination therapy with lenvatinib and doxorubicin remains largely unclear.

Materials and Methods: The antitumor efficacy of combination therapy with lenvatinib and doxorubicin on ATC cell proliferation was assessed by the MTT assay and colony formation. Flow cytometry was employed to assess ATC cells' apoptosis and cell cycle arrest in response to combination therapy. Transwell assay was used to test the migration and invasion in response to combination therapy. Xenograft models were used to test its in vivo antitumor activity.

Results: Lenvatinib monotherapy was less effective than doxorubicin in treating ATC cell lines and xenograft model. The combination therapy of lenvatinib and doxorubicin significantly inhibited ATC cell proliferation and tumor growth in nude mice, and induced cell apoptosis and cell cycle arrest as compared to lenvatinib or doxorubicin monotherapy.

Conclusion: Lenvatinib promotes the antitumor effect of doxorubicin in ATC cell and xenograft model. The lenvatinib/doxorubicin combination may be a potential candidate therapeutic approach for anaplastic thyroid cancer.

Keywords: anaplastic thyroid cancer, lenvatinib, doxorubicin, combining therapy

\section{Introduction}

ATC is the most aggressive type of thyroid cancer and one of the deadliest cancers in humans. ${ }^{1,2}$ The incidence of ATC accounts for less than $2 \%$ of thyroid cancer, but the mortality rate accounts for $15-40 \%$ of all thyroid cancers. ${ }^{3,4}$ ATC shows a rapid growth, easy metastasis and poor treatment outcomes, leading to a less than 10\% 5-year survival rate., ${ }^{2,5}$ The standard treatment for ATC includes surgery, debulking, external beam radiation therapy, chemotherapy and multisystemic therapy. ${ }^{6,7}$ Doxorubicin has been approved by the American Thyroid Association (ATA) guidelines in ATC, however, monotherapy still shows no improvement in the total survival in advanced ATC. ${ }^{6}$ Since combined therapy has shown a better response than monotherapy, ${ }^{8,9}$ therefore, there is an urgent need to find new multiple targets combined with therapies against ATC.

Lenvatinib is a multi-target tyrosine kinase inhibitor (MKI) that targets fibroblast growth factor receptors 1-4 (FGFR1-4), vascular endothelial growth factor receptors 1-3 (VEGFR1-3), c-KIT and RET. ${ }^{10}$ It has been approved by the FDA 
and EMA as the second-line treatment for advanced radioiodine-refractory differentiated thyroid carcinoma (DTC). ${ }^{11,12}$ A recent study indicated lenvatinib could significantly reduce cell growth and increase apoptosis of ATC cells both in vitro and in vivo. ${ }^{13}$ However, in a phase II study, postoperatively used lenvatinib to treat ATC patients indicated a response rate of only $17.4 \%{ }^{14}$ Besides, another phase II study mainly assessing the safety and efficacy of lenvatinib in patient with thyroid cancer, has reported a manageable safety profile while a modest effectiveness of lenvatinib in ATC treatment. ${ }^{15}$ These results indicated lenvatinib has a relatively low efficacy when administered as a monotherapy to treat ATC.

Although many studies have verified that doxorubicin or lenvatinib as a monotherapy have limited effectiveness, the combination of MKI with doxorubicin has shown increased anti-tumor activities in some cancers. ${ }^{16,17}$ However, the antitumor activities of combining doxorubicin with lenvatinib in ATC were unknown. Thus, we performed a series of in vitro and in vivo experiments to explore the antitumor effectiveness of the combination of lenvatinib and doxorubicin in ATC.

\section{Materials and Methods}

\section{Cell Culture and Drugs Administration}

ATC cell lines 8305C, 8505C and human immortalized thyroid epithelial cell Nthy-ori3-1 were kindly provided by Dr. Haixia Guan (The First Affiliated Hospital of China Medical University, Shenyang, P. R. China). C643 were obtained from Dr. Lei Ye (Ruijin Hospital, Shanghai, P. R. China). The use of the cell lines was approved by the Institutional Review Board of Xi'an Jiaotong University Health Science Center. All the cells were cultured in RPMI 1640 medium supplemented with $10 \%$ fetal bovine serum (Invitrogen Technologies, Inc., CA) at $37^{\circ}$ C. Cells were treated with lenvatinib (Selleck Chemicals, LLC) or/and doxorubicin (Selleck Chemicals, LLC) at the indicated concentrations and time points in some specific experiment. The lenvatinib and doxorubicin were dissolved in dimethylsulfoxide (DMSO), the same volume of DMSO was used as the vehicle control.

\section{Cell Viability Assay}

Cells (3000 to 4000/well) were seeded in 96-well plates. After a 24-h culture, cells were treated with indicated doses of doxorubicin or/and lenvatinib for the indicated period. Then $20 \mu \mathrm{L}$ of $5 \mathrm{mg} / \mathrm{mL}$ 3-(4,5-Dimethylthiazolyl- 2)-2,5-diphenyltetrazolium bromide (MTT) was employed to assess the cell viability as described. ${ }^{18}$ IC50 values were calculated with the Reed-Muench method. ${ }^{19}$

\section{Colony Formation Assay}

Monolayer culture was performed to measure colony formation. Cells (3000 to 4000/well) were seeded into 12-well plates, and cultured with various doses of doxorubicin and lenvatinib, individually or in combination (high doses for C643 because of its higher cells' density in one clone). The medium contains doxorubicin and lenvatinib, individually or in combination, was refreshed every $72 \mathrm{~h}$. After 7-12 days of cultivation, colonies were fixed with $4 \%$ paraformaldehyde, and then washed with PBS and stained with a crystal violet solution. Each assay was carried out in triplicate.

\section{Migration and Invasion Assay}

The indicated cells $\left(1 \times 10^{5}\right)$ were starved overnight and then seeded in the upper chamber of the transwell $(8.0 \mu \mathrm{m}$ pore size; Millipore, MA) in $200 \mu \mathrm{L}$ of medium containing $0.5 \%$ FBS and indicated concentration of doxorubicin or/and lenvatinib. The transwell chambers were pre-coated with Matrigel ( $4 \times$ dilution; $15 \mu \mathrm{L} /$ well; BD Bioscience, $\mathrm{NJ}$ ) for invasion assay. Medium with $20 \%$ FBS $(1 \mathrm{~mL})$ and indicated concentration of doxorubicin or/and lenvatinib was added to the lower chamber. After a $16 \mathrm{~h}$ (for $8305 \mathrm{C}$ and $8505 \mathrm{C}$ ) to 40 $\mathrm{h}$ (for C643) incubation, non-migrating/non-invading cells in the upper chamber were removed using a cotton swab, and migrating/invading cells were then fixed in $100 \%$ methanol and stained with crystal violet solution $(0.5 \%$ crystal violet in $2 \%$ ethanol). Photographs were taken randomly for five fields of each membrane. The number of migrating/invading cells was expressed as the average number of cells per microscopic field over five fields.

\section{Animal Studies}

$8505 \mathrm{C}\left(5 \times 10^{6}\right)$ cell lines were injected subcutaneously into the right armpit region of 5- to 6-week-old female nude mice purchased from SLAC laboratory Animal Co., Ltd. (Shanghai, China) to establish xenograft mouse model. Mice were then randomly divided into four groups (3 mice each group) when tumor volume grew to $50-80 \mathrm{~mm}^{3}$. Doxorubicin (3 $\mathrm{mg}$ per kg of body weight) was administered by intraperitoneal injection at a total volume of $0.2 \mathrm{~mL}$ every day, and/or lenvatinib ( $5 \mathrm{mg}$ per $\mathrm{kg}$ of body weight) was administered via oral route every day. The treatment was administered for 3 weeks. All mice were sacrificed via cervical dislocation, and tumors were collected and 
weighted $5 \mathrm{~h}$ after the final dose of drugs. Animal experiments were approved by the Institutional Review Board of Xi'an Jiaotong University Health Science Center and followed the "National Institutes of Health Guide for the Care and Use of Laboratory Animals".

\section{Immunohistochemical (IHC) Staining}

Tumor tissues were embedded in paraffin, sectioned at $4 \mu \mathrm{m}$, then cell proliferation ability was assessed by quantification of Ki-67 staining (percentage of positive cells). In brief, antihuman Ki-67 antibody (BD Pharmingen, CAT 550609) and p-ERK (Cell Signaling Technology, CAT\#4370) were 1:300 diluted, and immunostaining was done according to a standard protocol using DAB Substrate Kit (ZSGB-BIO). To ensure the comparability of immunohistochemical staining, a common reference standard was included as an internal or intra-assay control in each batch. Ki-67 protein expression was scored with $0,1,2$, and 3 , which represent negative, weak positive, positive, and strong positive, respectively. Ki67 protein expression scored with 1, 2, and 3 were calculated as positive, the percent of positive staining cells were then calculated. ERK phosphorylation was quantitated by integral optical density (IOD) using Image-pro plus 6.0 (Media Cybernetics, USA). Each stained section was evaluated under the same magnification, light brightness, and exposure intensity. To evaluate the effect of different treatments on different organs, we performed hematoxylin and eosin (H\&E) staining of liver, kidney, and heart sections.

\section{Statistical Analysis}

All the statistical analyses were performed with the SPSS statistical package (16.0, SPSS Inc. Chicago, IL). Unpaired Student's $t$-test was used to compare the means of two groups of data. One-way analysis of variance (ANOVA) followed by Bonferroni's multiple comparison test was used to compare differences between three or more groups. All values were expressed as the mean \pm standard deviation (SD). $P<0.05$ was considered as a statistically significant difference. Each experiment was carried out and calculated in triplicate.

\section{Results}

\section{Lenvatinib Potentiates}

Proliferation-Inhibitory Effects of Doxorubicin in ATC Cells

Doxorubicin has shown encouraging clinical activity in systematic therapy of ATC. ${ }^{6}$ Thus, we first confirmed the proliferation inhibitory effects of doxorubicin in three ATC cell lines: 8305C, C643 and 8505C. As shown in Figure 1A, doxorubicin significantly inhibited the proliferation of ATC cells in a dose-dependent manner, with the IC50 values ranged from 5.84 to $13.31 \mathrm{nM}$. And immortalized thyroid epithelial cell Nthy-ori3-1 cells was not sensitive with doxorubicin (Figure 1A). Since we have proved the antitumor activities of lenvatinib towards ATC cells, ${ }^{8}$ we next tested the effect of a combination of lenvatinib and doxorubicin on the proliferation of ATC cells. As it showed in Figure 1B, the combination of lenvatinib $(1 \mu \mathrm{M})$ and doxorubicin $(10 \mathrm{nM})$ indicated a synergistic inhibitory effect on proliferation in ATC cells as compared to either monotherapy. Moreover, the viability of Nthy-ori3-1 cells was almost unaffected (Figure 1B).

\section{The Combination of Lenvatinib and Doxorubicin Synergistically Inhibits Colony Formation in Thyroid Cancer Cells}

Next, the growth-inhibitory effect of the combination of lenvatinib and doxorubicin was further explored with colony formation assay. As expected, lenvatinib as well as doxorubicin applied alone at the indicated concentrations showed the colony forming inhibitory effect in monolayer cultured ATC cell lines-8305C, C643 and 8505C (Figure 2A-C). Moreover, a combination of lenvatinib and doxorubicin caused a strongly enhanced inhibition of ATC cells' colony formation as compared to doxorubicin monotherapy (Figure 2A-C). Collectively, these data suggested that the combination of doxorubicin with lenvatinib may greatly enhance the tumor suppression function of doxorubicin.

\section{Lenvatinib Potentiates Migration and Invasion-Inhibitory Effects of Doxorubicin in ATC Cells}

Migration and invasion are the main features of cancer cells and the major cause of ATC related death. ${ }^{20-22}$ Thus, we performed a transwell assay to investigate the contribution of lenvatinib and doxorubicin, individually or in combination, on ATC cell migration and invasion. Compared with the vehicle control, both lenvatinib and doxorubicin inhibited the migration of $8305 \mathrm{C}$ and $8505 \mathrm{C}$ cells at the indicated concentrations, while lenvatinib monotherapy shows no significance for C643 cells (Figure 3A-C). Moreover, 

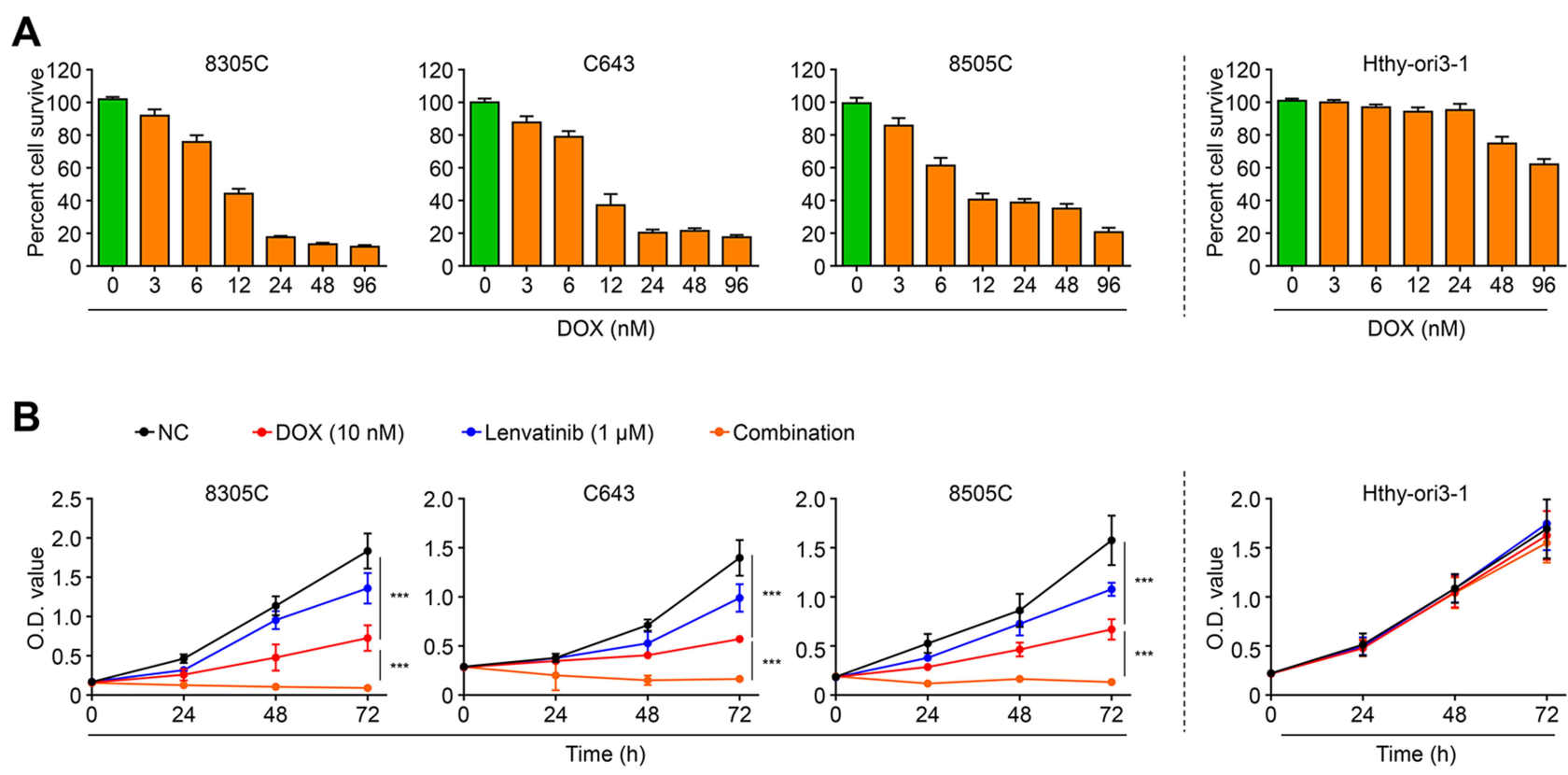

Figure I The combination of lenvatinib and doxorubicin inhibited the proliferation of different ATC cells. (A) Cells were treated with different concentrations of doxorubicin for $48 \mathrm{~h}$. MTT assay was employed to evaluate cell viability. (B) MTT assay was used to evaluate the proliferation inhibitory effect of combining therapy. Data were presented as mean \pm SD. $* * * P<0.001$.

A
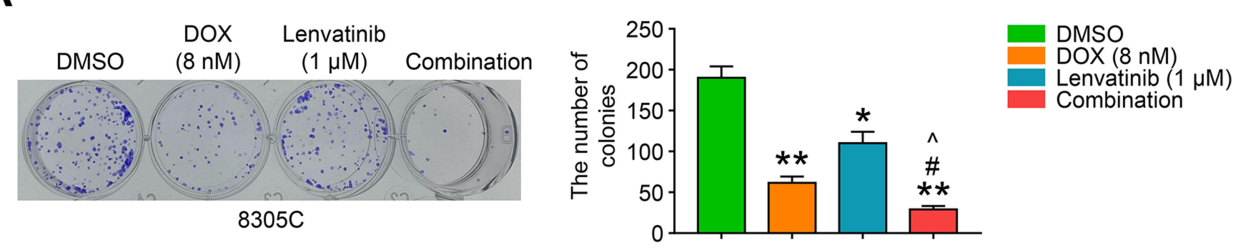

B
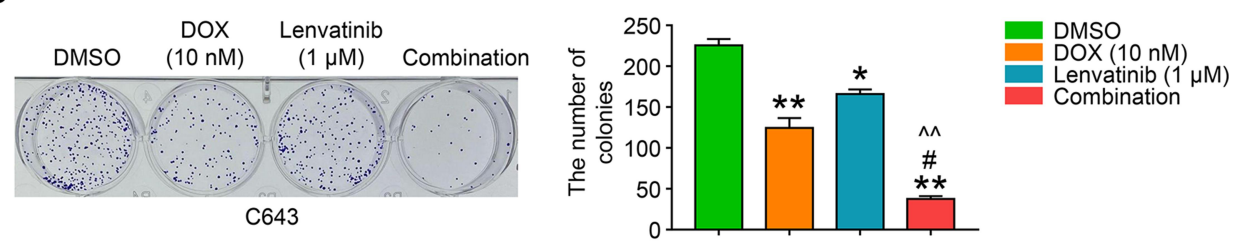

C
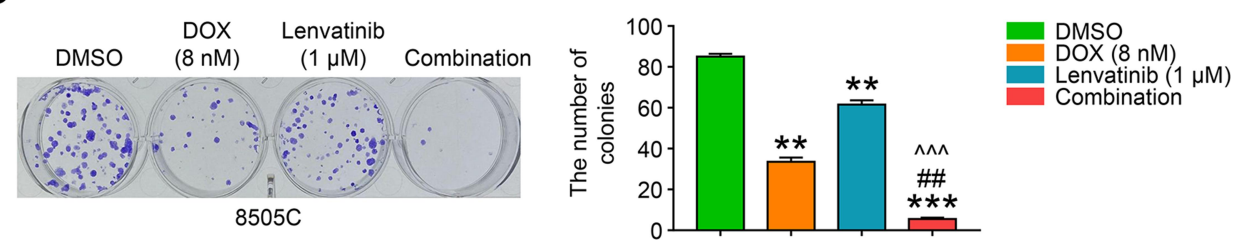

Figure 2 The combination of lenvatinib and doxorubicin inhibited the colony formation of different ATC cells. Representative figures of colony formation in 8305C (A), $\mathrm{C} 643$ (B) and $8505 \mathrm{C}$ (C) cells were shown in the left panel. The number of colonies was calculated and presented in right panel. Data were presented as mean \pm SD. ${ }^{*} \#, \wedge<$ $0.05 ;{ }^{* *}, \#, \wedge \wedge<0.01 ;{ }^{* * * k, \wedge \wedge} P<0.001$. *Compared with DMSO group; ${ }^{*}$ Compared with DOX group; 'Compared with Lenvatinib group.

addition of lenvatinib to doxorubicin synergistically decreased the migrated cells in comparison to either lenvatinib or doxorubicin monotherapy (Figure 3A-C). Besides, the addition of lenvatinib to doxorubicin induced less invaded cells among three ATC cell lines compared with the vehicle control or monotherapy (Figure 4A-C). 
A
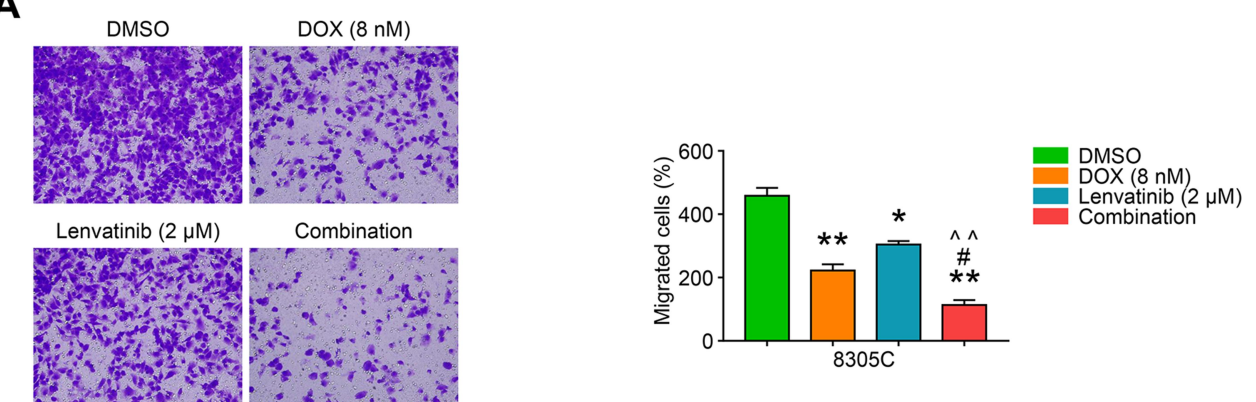

B

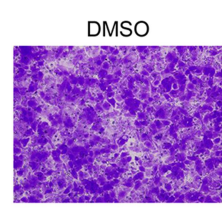

DOX (15 nM)

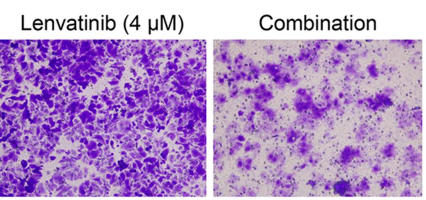

C

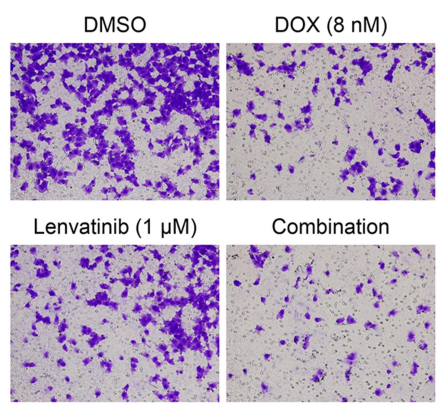

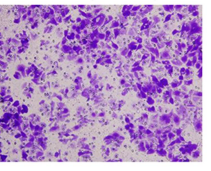

\section{.}

\section{.}

\section{.} . (1)

Figure 3 The combination of lenvatinib and doxorubicin synergistically inhibited the migration of ATC cells. $8305 \mathrm{C}$ (A), C643 (B) and 8505C (C) were treated with lenvatinib or/and doxorubicin with the indicated concentration for 48-72 h. Representative figures showed the migrated cells in the chamber (left panel, 200 times). Quantitative illustration of migrated cells was shown in right panel. Data were presented as mean $\pm \mathrm{SD}$. ${ }^{*, \#, \wedge} P<0.05 ;{ }^{* *, \wedge} P<0.01$. ${ }^{*}$ Compared with $\mathrm{DMSO}$ group; \#Compared with DOX group; 'Compared with Lenvatinib group.

Abbreviation: ns, no statistical significance.

\section{Lenvatinib Potentiates Antitumor Effect of Doxorubicin in vivo}

To determine whether the enhanced antitumor effect of doxorubicin by the addition of lenvatinib can also be observed in vivo, we established $8505 \mathrm{C}$ cell derived xenograft tumor in nude mice. These mice were treated with lenvatinib and doxorubicin, individually or in combination at the indicated concentrations and time points. As shown in Figure $5 \mathrm{~A}$, the tumor weight of $8505 \mathrm{C}$ cell-derived xenograft tumors in the monotherapy groups were lighter than that in vehicle group; and the combination group was much lighter than that in monotherapy groups. Moreover, we assessed the cell proliferation in tumor tissues by IHC staining Ki-67. The number of Ki-67 positive cells in tumors from combination group was lower than that in monotherapy groups; while the monotherapy groups showed lower Ki-67 staining as compared to the vehicle control (Figure 5B). Besides, histopathological evidences also showed that the combination therapy did not cause more significant organ injury than that of monotherapy in mice (Figure 5C). To further identify the mechanism underlying the combination therapy, we examine the phosphorylation of ERK, which is the downstream of VEGFR inhibiting by lenvatinib,${ }^{10}$ through IHC. The phosphorylation of ERK in the combination group was weaker than that in the monotherapy groups, indicating the synergistic effect enhanced the function of both drugs (Figure 6). As a result, our data demonstrated the efficacy and safety of the combination of lenvatinib and doxorubicin for ATC treatment. 
A

A

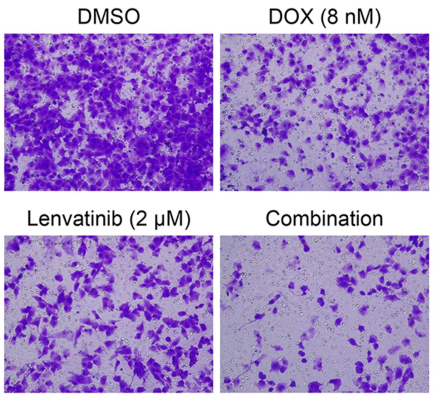

B

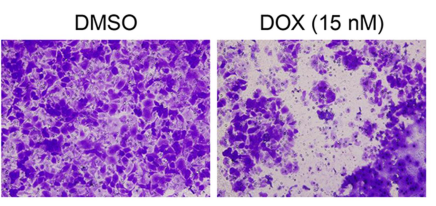

Lenvatinib $(4 \mu \mathrm{M})$

Combination
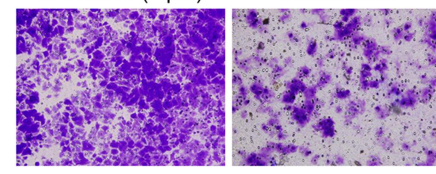

C
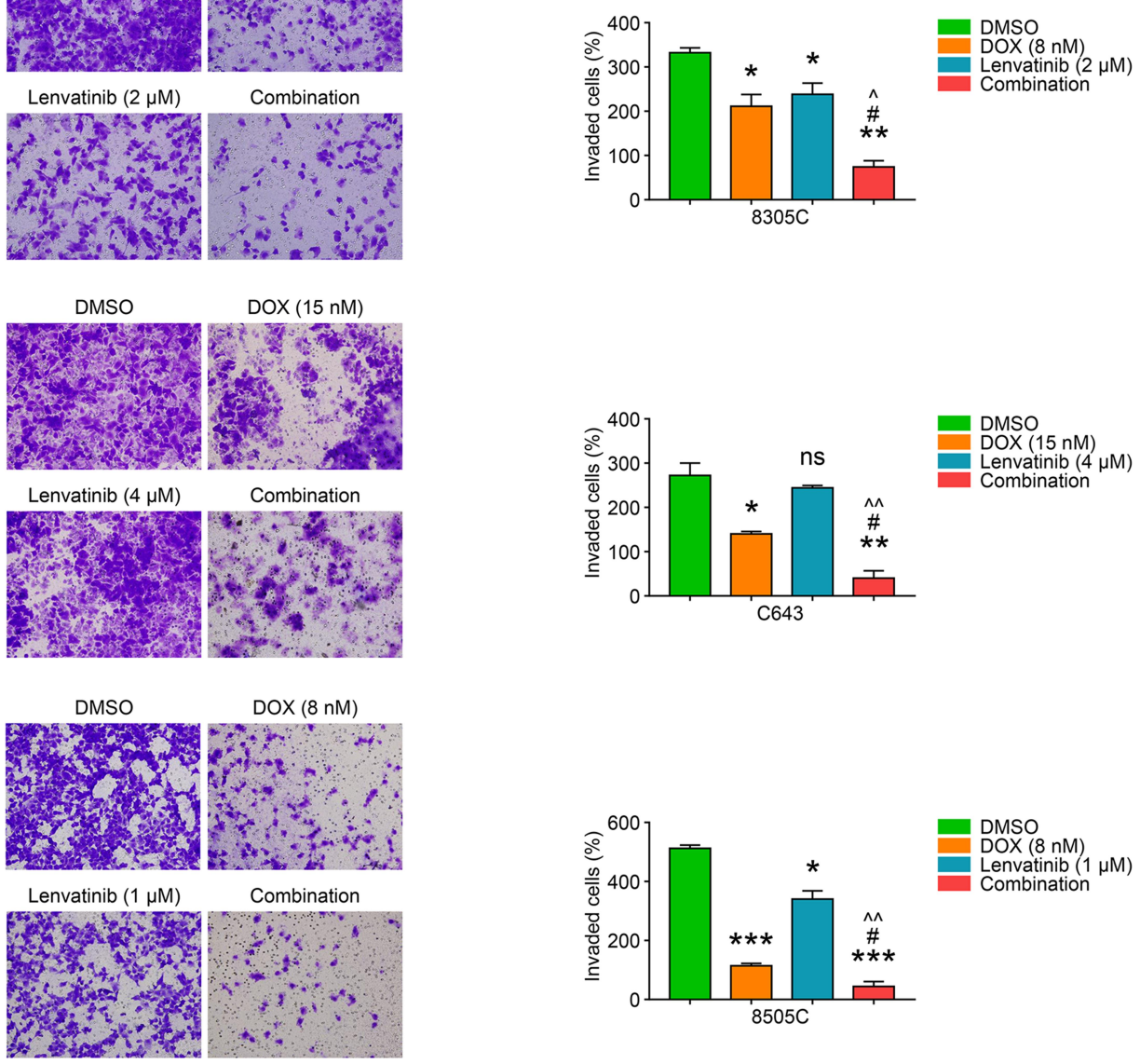

Figure 4 The combination of lenvatinib and doxorubicin synergistically inhibited the invasion of ATC cells. 8305C (A), C643 (B) and 8505C (C) were treated with lenvatinib or/and doxorubicin with the indicated concentration for 48-72 h. Representative figures showed the invaded cells (left panel, 200 times). Quantitative analysis of invaded cells was shown in right panel. Data were presented as mean \pm SD. ${ }^{*, \#,} P<0.05 ;{ }^{* * \wedge \wedge} P<0.01$; ***P < 0.001 . ${ }^{*}$ Compared with DMSO group; ${ }^{\#}$ Compared with DOX group; ^Compared with Lenvatinib group.

Abbreviation: ns, no statistical significance.

\section{Discussion}

ATC belongs to the undifferentiated thyroid cancer type and its incidence accounts for less than $2 \%$ among the thyroid cancers. However, the mortality rate of ATC remains high due to its easy metastasis and therapy resistance. $^{5}$ Surgical resection of the solid tumor, assisted with systemic adjuvant therapy, is the main therapy for ATC. However, the mainstay approaches were met with limited effectiveness in some patients. ${ }^{23}$ Indeed, with the development of molecular characterization exploring in ATC, researchers have gained a better understanding of its molecular pathogenesis in recent years. ${ }^{24}$ These drive more novel treatments for anaplastic thyroid carcinoma. ${ }^{12}$ Some of the new biological agents have shown potential in ATC treatment. For example, ATC was found to markedly express PD-L1 than DTC.
Therefore, combining PD-L1 antibody with BRAF inhibitor PLX4720 or other kinase inhibitors could be used in ATC treatment. ${ }^{25,26}$ In a Phase I trial, three patients with ATC achieved complete remission and survived for more than 30 months with the administration of combretastatin A-4 phosphate. ${ }^{27}$ Another clinical study showed the complete response in a 49 year old women ATC patient by chemoradiation with dabrafenib. ${ }^{28}$ These observations suggested that antitumor activity of the new potential agents against ATC were promising.

Nowadays, small molecule inhibitors targeting multiple tyrosine kinases have attracted great attention because of their clinical benefits in ATC treatment. ${ }^{12}$ Therefore, some of them have been approved by the US FDA for management of different cancers including thyroid cancer such as vandetanib and lenvatinib, and some of them are in the 
A

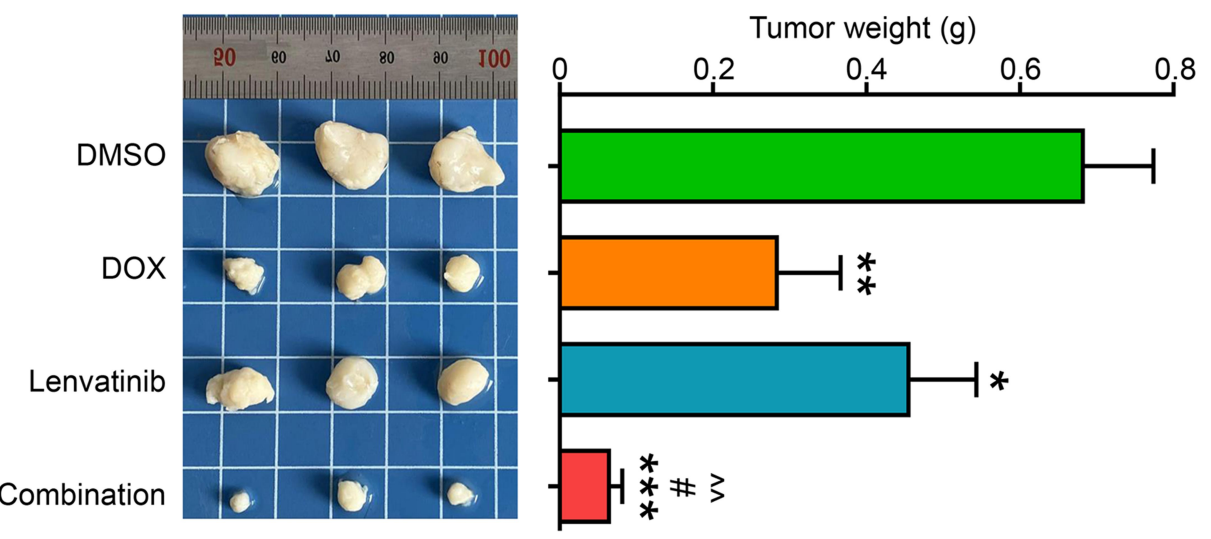

B
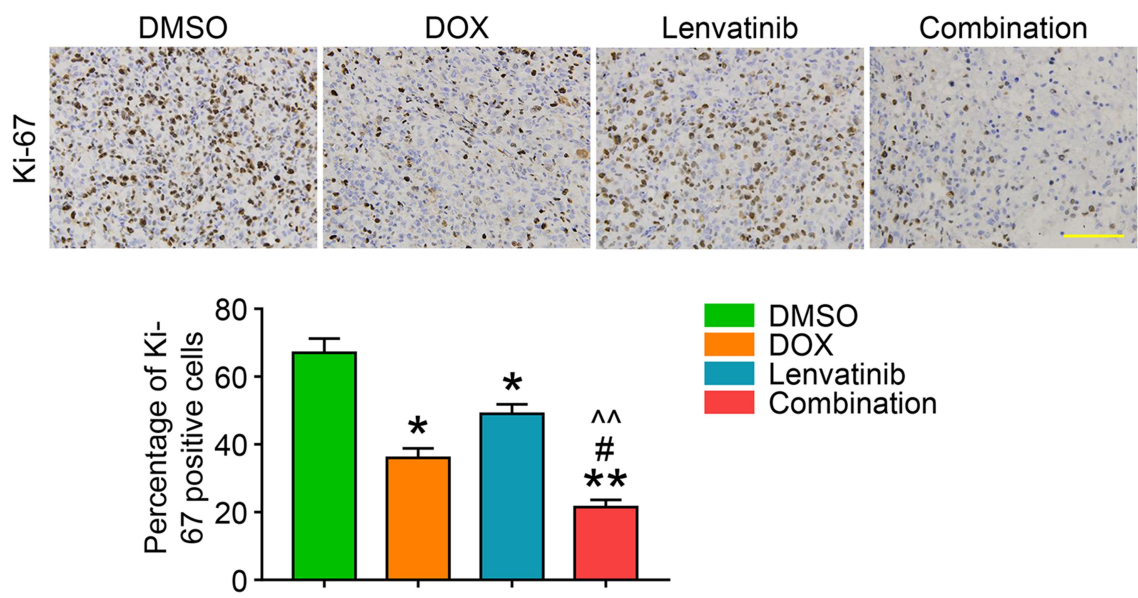

C

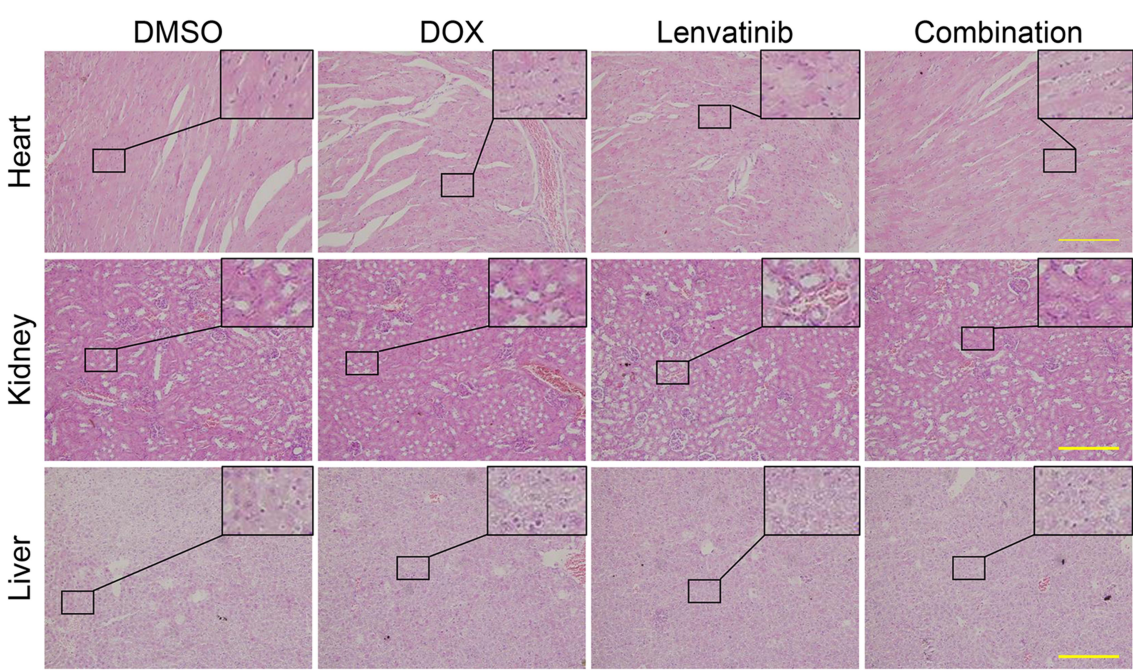

Figure 5 Validation of combining lenvatinib and doxorubicin as a functional therapy in vivo. (A) Representative image showed the isolated tumors from mice in different groups (left panel), and the bar chart illustrated the tumor weight. (B) The levels of Ki-67 proteins in the indicated xenograft tumors were measured by IHC assay (upper panels). Statistical analysis of the percentage of Ki-67 positive cells was shown in lower panels. Scale bars, $200 \mu \mathrm{m}$. (C) Representative H\&E stained heart, kidney and liver sections from the indicated mice. Scale bars, $200 \mu \mathrm{m}$. Data were presented as mean \pm SD. ${ }^{* \#} P<0.05 ;{ }^{* * \wedge \wedge} P<0.01 ;{ }^{* * * P}<0.00$ I. ${ }^{*}$ Compared with DMSO group; ${ }^{\#}$ Compared with DOX group; ${ }^{\wedge}$ Compared with Lenvatinib group. 

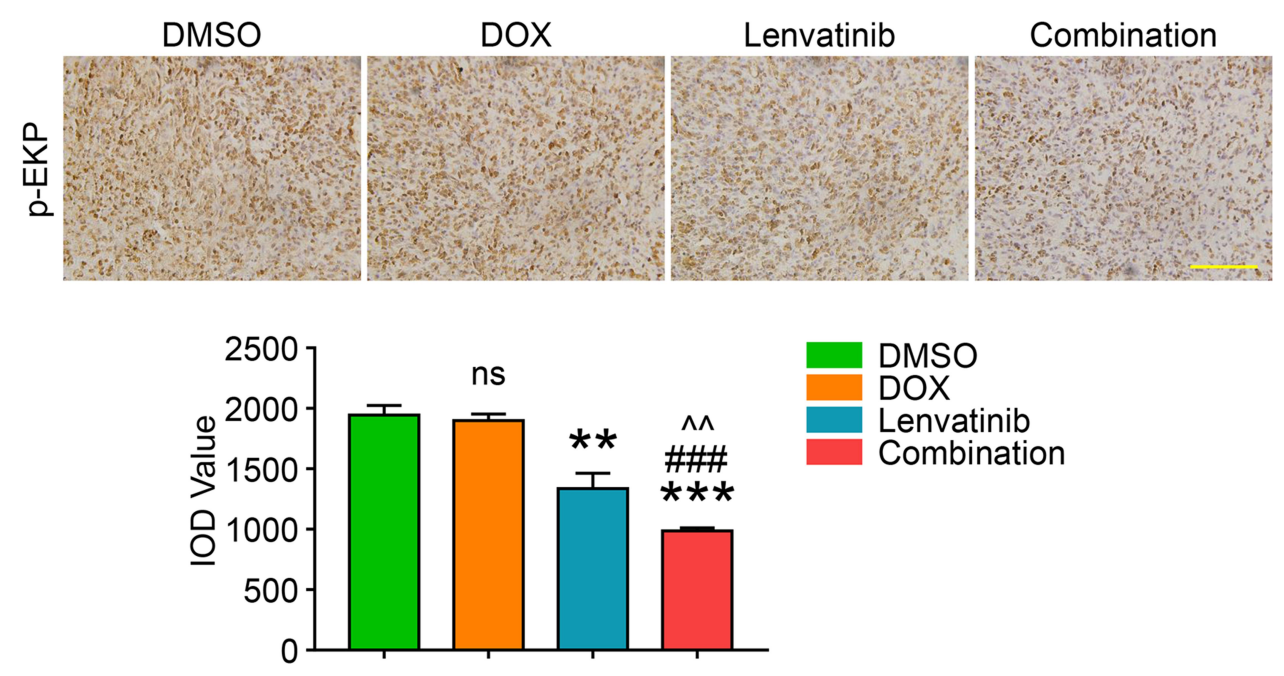

Figure 6 Identification of the mechanism underlying combining lenvatinib and doxorubicin as a functional therapy in vivo. Representative image showed the levels of ERK phosphorylation in the indicated xenograft tumors, which were measured by IHC assay (upper panels). Statistical analysis of the IOD value was shown in lower panels. Scale bars, $200 \mu \mathrm{m}$. Data were calculated from 3 different views and presented as mean \pm SD. ${ }^{* *, \wedge} P<0.0$ I; ${ }^{* * *}$, $P<0.00$ I. ${ }^{*}$ Compared with DMSO group; ${ }^{*}$ Compared with DOX group; ^Compared with Lenvatinib group.

process of clinical trials. ${ }^{12}$ The oral MKI lenvatinib was approved by the FDA in treating radioiodine refractory DTC, and some studies also indicated its modest antitumor effect in ATC patients. However, the usage dose should be reduced owing to the grade 3 or higher adverse event. $^{12,14,15}$ These results suggested that lenvatinib monotherapy has limited clinical activity in ATC. Doxorubicin was approved by ATA guidelines as a conventional singleagent in ATC treatment, ${ }^{6}$ and some kind of MKIs was proved to enhance the anti-tumor effect of doxorubicin in different cancers. ${ }^{29,30}$ We thus investigated the feasibility of the combination of lenvatinib and doxorubicin for ATC treatment.

Our data indicated that lenvatinib did show some effectiveness but less than that of doxorubicin in ATC cell lines and xenografts. Notably, we found that lenvatinib enhanced the effects of doxorubicin in ATC treatment both in vitro and in vivo. The combination of lenvatinib and doxorubicin synergistically inhibited ATC cells' proliferation and colony formation. Moreover, combining lenvatinib with doxorubicin potentiated the migration and invasion inhibition compared with its monotherapy. Importantly, the dose of both lenvatinib and doxorubicin in combinatorial therapies did not show additional side effects such as cardiotoxicity compared to either monotherapy. These findings suggested a potential combination therapy in the management of ATC.

Actually, combining lenvatinib with conventional or newly developed anti-cancer drugs has been investigated for potential therapies. ${ }^{8,31}$ Our data demonstrated that lenvatinib enhanced the antitumor effects of conventional anti-cancer drug doxorubicin in ATC not only in vitro but also in vivo. Combining with our previous study, ${ }^{8}$ we supposed that lenvatinib may be able to enhance the antitumor ability of a panel of anti-tumor drugs, which mainly targeted mitosis. These may be due to the inhibition of angiogenesis by lenvatinib, which will enhance the energy deficiency in mitosis. ${ }^{32}$ The mitogen-activated protein kinase (MAPK) pathway has been proved to play an important role in DNA repair in response to DNA damage. ${ }^{33,34}$ Consistent with our result, the inhibition of receptor tyrosine kinases (RTKs) and its downstream by lenvatinib impaired the ability of DNA repair, and combined with lenvatinib will enhance the DNA damaging by doxorubicin. However, further investigations into the detailed molecular mechanisms are still needed. In summary, these preliminary results indicated that the lenvatinib/doxorubicin combination therapy may be an encouraging treatment for ATC.

\section{Conclusion}

Our data demonstrate that lenvatinib enhances the antitumor effect of doxorubicin in ATC. Lenvatinib/doxorubicin synergy was confirmed not only in vitro but also in vivo. These encouraging preliminary results suggest that lenvatinib/doxorubicin combination may be a potential therapeutic approach for ATC. 


\section{Ethics Approval and Informed Consent}

The Ethics Committee of The First Affiliated Hospital of Xi'an Jiaotong University approved the using of cell lines in this research. And the animal experiments were performed according to the guidelines for use and care of animals approved by The First Affiliated Hospital of Xi'an Jiaotong University.

\section{Acknowledgments}

We thank Dr. Haixia Guan (The First Affiliated Hospital of China Medical University, Shenyang, China) and Lei Ye (Ruijin Hospital, Shanghai, China) for kindly providing human thyroid cancer cell lines.

\section{Author Contributions}

XS performed all the experimental work. QQG and HHZ participated in data analysis. DMY and MJJ conceived and participated in the design of the study. The manuscript was written by XS, XRZ and MJJ. All authors contributed to data analysis, drafting, or revising the article, have agreed on the journal to which the article will be submitted, gave final approval of the version to be published, and agree to be accountable for all aspects of the work.

\section{Funding}

This work was supported by the Key Research and Development Projects of Shaanxi Province (No.2019SF022).

\section{Disclosure}

The authors declare no competing interests.

\section{References}

1. Siegel RL, Miller KD, Jemal A. Cancer statistics, 2015. CA Cancer J Clin. 2015;65(1):5-29. doi:10.3322/caac.21254

2. Are C, Shaha AR. Anaplastic thyroid carcinoma: biology, pathogenesis, prognostic factors, and treatment approaches. Ann Surg Oncol. 2006;13(4):453-464. doi:10.1245/ASO.2006.05.042

3. Siegel RL, Miller KD, Jemal A. Cancer statistics, 2019. CA Cancer J Clin. 2019;69(1):7-34. doi:10.3322/caac.21551

4. Kitamura Y, Shimizu K, Nagahama M, et al. Immediate causes of death in thyroid carcinoma: clinicopathological analysis of 161 fatal cases. J Clin Endocrinol Metab. 1999;84(11):4043-4049. doi:10.1210/ jcem.84.11.6115

5. Kebebew E, Greenspan FS, Clark OH, Woeber KA, McMillan A. Anaplastic thyroid carcinoma. Treatment outcome and prognostic factors. Cancer. 2005;103(7):1330-1335. doi:10.1002/cncr.20936

6. Smallridge RC, Ain KB, Asa SL, et al. American thyroid association guidelines for management of patients with anaplastic thyroid cancer. Thyroid. 2012;22(11):1104-1139. doi:10.1089/thy.2012.0302
7. Ito K, Hanamura T, Murayama K, et al. Multimodality therapeutic outcomes in anaplastic thyroid carcinoma: improved survival in subgroups of patients with localized primary tumors. Head Neck. 2012;34(2):230-237. doi:10.1002/hed.21721

8. Jing C, Gao Z, Wang R, Yang Z, Shi B, Hou P. Lenvatinib enhances the antitumor effects of paclitaxel in anaplastic thyroid cancer. $A m$ J Cancer Res. 2017;7(4):903-912.

9. Yau T, Lo CY, Epstein RJ, Lam AK, Wan KY, Lang BH. Treatment outcomes in anaplastic thyroid carcinoma: survival improvement in young patients with localized disease treated by combination of surgery and radiotherapy. Ann Surg Oncol. 2008;15(9):2500-2505. doi:10.1245/s10434-008-0005-0

10. Cabanillas ME, Habra MA. Lenvatinib: role in thyroid cancer and other solid tumors. Cancer Treat Rev. 2016;42:47-55. doi:10.1016/j. ctrv.2015.11.003

11. Schlumberger M, Jarzab B, Cabanillas ME, et al. A phase II trial of the multitargeted tyrosine kinase inhibitor lenvatinib (E7080) in advanced medullary thyroid cancer. Clin Cancer Res. 2016;22 (1):44-53. doi:10.1158/1078-0432.CCR-15-1127

12. Ferrari SM, Elia G, Ragusa F, et al. Novel treatments for anaplastic thyroid carcinoma. Gland Surg. 2020;9(Suppl 1):S28-S42. doi: $10.21037 /$ gs.2019.10.18

13. Ferrari SM, Bocci G, Di Desidero T, et al. Lenvatinib exhibits antineoplastic activity in anaplastic thyroid cancer in vitro and in vivo. Oncol Rep. 2018;39(5):2225-2234. doi:10.3892/ or.2018.6306

14. Iwasaki H, Yamazaki H, Takasaki H, et al. Lenvatinib as a novel treatment for anaplastic thyroid cancer: a retrospective study. Oncol Lett. 2018;16(6):7271-7277. doi:10.3892/ol.2018.9553

15. Takahashi S, Kiyota N, Yamazaki T, et al. A phase II study of the safety and efficacy of lenvatinib in patients with advanced thyroid cancer. Future Oncol. 2019;15(7):717-726. doi:10.2217/fon-20180557

16. Estupinan O, Santos L, Rodriguez A, et al. The multikinase inhibitor EC-70124 synergistically increased the antitumor activity of doxorubicin in sarcomas. Int $J$ Cancer. 2019;145(1):254-266. doi:10.1002/ ijc. 32081

17. Badinloo M, Esmaeili-Mahani S. Phosphatidylinositol 3-kinases inhibitor LY294002 potentiates the cytotoxic effects of doxorubicin, vincristine, and etoposide in a panel of cancer cell lines. Fundam Clin Pharmacol. 2014;28(4):414-422. doi:10.1111/fcp.12043

18. Su X, Shen Z, Yang Q, et al. Vitamin C kills thyroid cancer cells through ROS-dependent inhibition of MAPK/ERK and PI3K/AKT pathways via distinct mechanisms. Theranostics. 2019;9 (15):4461-4473. doi:10.7150/thno.35219

19. Welkos S, O'Brien A. Determination of median lethal and infectious doses in animal model systems. Methods Enzymol. 1994;235:29-39. doi:10.1016/0076-6879(94)35128-7

20. Hanahan D, Weinberg RA. Hallmarks of cancer: the next generation. Cell. 2011;144(5):646-674. doi:10.1016/j.cell.2011.02.013

21. Hanahan D, Weinberg RA. The hallmarks of cancer. Cell. 2000;100 (1):57-70. doi:10.1016/s0092-8674(00)81683-9

22. Smallridge RC, Marlow LA, Copland JA. Anaplastic thyroid cancer: molecular pathogenesis and emerging therapies. Endocr Relat Cancer. 2009;16(1):17-44. doi:10.1677/ERC-08-0154

23. Keutgen XM, Sadowski SM, Kebebew E. Management of anaplastic thyroid cancer. Gland Surg. 2015;4(1):44-51. doi:10.3978/j. issn.2227-684X.2014.12.02

24. Landa I, Ibrahimpasic T, Boucai L, et al. Genomic and transcriptomic hallmarks of poorly differentiated and anaplastic thyroid cancers. J Clin Invest. 2016;126(3):1052-1066. doi:10.1172/ JCI85271

25. Iyer PC, Dadu R, Gule-Monroe M, et al. Salvage pembrolizumab added to kinase inhibitor therapy for the treatment of anaplastic thyroid carcinoma. J Immunother Cancer. 2018;6(1):68. doi:10.1186/s40425-018-0378-y 
26. Brauner E, Gunda V, Vanden Borre P, et al. Combining BRAF inhibitor and anti PD-L1 antibody dramatically improves tumor regression and anti tumor immunity in an immunocompetent murine model of anaplastic thyroid cancer. Oncotarget. 2016;7 (13):17194-17211. doi:10.18632/oncotarget.7839

27. Dowlati A, Robertson K, Cooney M, et al. A phase I pharmacokinetic and translational study of the novel vascular targeting agent combretastatin a-4 phosphate on a single-dose intravenous schedule in patients with advanced cancer. Cancer Res. 2002;62(12):3408-3416.

28. Lim AM, Taylor GR, Fellowes A, et al. BRAF inhibition in BRAFV600E-positive anaplastic thyroid carcinoma. J Natl Compr Canc Netw. 2016;14(3):249-254. doi:10.6004/jnccn.2016.0030

29. Aggerholm-Pedersen N, Demuth C, Safwat A, Meldgaard P, Kassem M, Sandahl Sorensen B. Dasatinib and doxorubicin treatment of sarcoma initiating cells: a possible new treatment strategy. Stem Cells Int. 2016;2016:9601493. doi:10.1155/2016/9601493

30. Chen W, Liu I, Tomiyasu H, et al. Imatinib enhances the anti-tumour effect of doxorubicin in canine B-cell lymphoma cell line. Vet J. 2019;254:105398. doi:10.1016/j.tvj1.2019.105398
31. Chen WX, Li GX, Hu ZN, Zhu P, Zhang BX, Ding ZY. Significant response to anti-PD-1 based immunotherapy plus lenvatinib for recurrent intrahepatic cholangiocarcinoma with bone metastasis: a case report and literature review. Medicine (Baltimore). 2019;98 (45):e17832. doi:10.1097/MD.0000000000017832

32. Clapier CR, Cairns BR. The biology of chromatin remodeling complexes. Annu Rev Biochem. 2009;78:273-304. doi:10.1146/ annurev.biochem.77.062706.153223

33. Wood CD, Thornton TM, Sabio G, Davis RA, Rincon M. Nuclear localization of $\mathrm{p} 38 \mathrm{MAPK}$ in response to DNA damage. Int J Biol Sci. 2009;5(5):428-437. doi:10.7150/ijbs.5.428

34. Ulm R, Revenkova E, Di Sansebastiano GP, Bechtold N, Paszkowski J. Mitogen-activated protein kinase phosphatase is required for genotoxic stress relief in Arabidopsis. Genes Dev. 2001;15(6):699-709. doi:10.1101/Gad.192601

\section{Publish your work in this journal}

OncoTargets and Therapy is an international, peer-reviewed, open access journal focusing on the pathological basis of all cancers, potential targets for therapy and treatment protocols employed to improve the management of cancer patients. The journal also focuses on the impact of management programs and new therapeutic agents and protocols on patient perspectives such as quality of life, adherence and satisfaction. The manuscript management system is completely online and includes a very quick and fair peer-review system, which is all easy to use. Visit http://www.dovepress.com/ testimonials.php to read real quotes from published authors. 